\title{
EFECTO DE VARIEDADES Y DENSIDAD DE PLANTACIÓN EN LA CALIDAD FÍSICA DEL FLORETE DE BRÓCOLI (Brassica oleracea var. italica)
}

\section{EFFECT OF VARIETIES AND PLANT DENSITY ON THE PHYSICAL QUALITY OF BROCCOLI (Brassica oleracea var. italica)}

\author{
Ma. de Lourdes Fraire Cordero ${ }^{1}$, Daniel Nieto Ángel ${ }^{1 *}$, Elizabeth Cárdenas Soriano ${ }^{1}$, Gabriel Gutiérrez \\ Alonso $^{2}$, Rafael Bujanos Muñiz ${ }^{3}$ y Humberto Vaquera Huerta ${ }^{4}$
}

\begin{abstract}
${ }^{1}$ Instituto de Fitosanidad, Colegio de Postgraduados, Campus Montecillo. Km. 36.5 Carr. México-Texcoco. 56230, Montecillo, Texcoco, Edo. de México. Tel. 0159520200 Ext. 1626. 'Estación Experimental "La Charca”. A 3 kms del kilometro 11 de la carretera Cortazar-Jaral del Progreso, 38470. Jaral del Progreso, Guanajuato. ${ }^{3}$ Instituto Nacional de Investigaciones Forestales, Agricolas y Pecuarias. Km. 6.5 Carr. Celaya-San Miguel de Allende, 38010, Celaya, Guanajuato. ${ }^{4}$ Instituto de Socioeconomía, Estadística e Informática, Colegio de Postgraduados, Campus Montecillo. Km. 36.5 Carr. MéxicoTexcoco. 56230, Montecillo, Texcoco, Edo. de México.
\end{abstract}

* Autor para correspondencia (dnieto@colpos.mx)

\section{RESUMEN}

En 2005 y 2006 se evaluaron ocho y doce cultivares comerciales de brócoli respectivamente, en Apaseo el Grande, Guanajuato, México, en tres densidades de plantación $\left(55,65,75\right.$ mil plantas ha $\left.{ }^{-1}\right)$, en parcelas de $48 \mathrm{~m}^{2}$. Al momento de la cosecha se midió diámetro del florete, diámetro del hueco en el tallo y número de minifloretes, y se registró la pérdida de peso a temperatura ambiente $\left(18-25^{\circ} \mathrm{C}\right)$ en las $40 \mathrm{~h}$ posteriores a la cosecha. Los resultados mostraron que la densidad de plantación tiene un efecto inversamente proporcional al diámetro de florete y al hueco en el tallo. La densidad de 55000 plantas ha ${ }^{-1}$ produjo mayor diámetro de florete $y$ de hueco en el tallo, mientras que la densidad de 75000 plantas ha ${ }^{-1}$. El cultivar 'Monaco' presentó el mayor diámetro de florete, 'Ironman' tuvo el menor diámetro de hueco en el tallo. El número de minifloretes no estuvo influenciado por la densidad de plantación, pero sí por el cultivar ya que las vars. 'Grandísimo' y 'Avenger' superaron a los demás en cantidad de minifloretes. La menor pérdida de peso se registró en floretes a una densidad de plantación de 65000 plantas ha $^{-1}$.

Palabras clave: Brassica oleracea, senescencia, postcosecha.

\section{SUMMARY}

Eight and twelve broccoli cultivars were evaluated respectively, during 2005 and 2006, in Apaseo el Grande, Guanajuato, México. They were transplanted in three plant densities $(55,65,75$ thousands plants ha-1) in $48 \mathrm{~m}^{2}$ plots. At harvest, floret diameter, diameter of the stem hollow, and number of branchlets were recorder, while ambient temperature weight loss $\left(18-25^{\circ} \mathrm{C}\right)$ was followed during 40 $h$ after harvest. Results showed that density has an inversely proportional effect on floret diameter and stem hollow. At 55000 plants $\mathrm{ha}^{-1}$ floret diameter and hollows reached their peak while the 75000 plants ha ${ }^{-1}$ density produced the smallest floret diameter. $\mathrm{Cv}$. 'Monaco' had the largest floret diameter whereas cv. 'Ironman' presented the smallest hollow diameter. Branchlet number was not influenced by plant density yet it depended on variety. Cvs 'Grandísimo' and 'Avenger' had the largest number of branchlets.
The smallest weight loss was registered in florets from a plant density of 65000 plants ha- ${ }^{-1}$.

Index words: Brassica oleracea, senescence, postharvest.

\section{INTRODUCCIÓN}

El brócoli (Brassica oleracea L.) es una hortaliza de importancia económica a nivel mundial debido a sus valores alimenticios y medicinales. Tanto las hojas como la inflorescencia (florete) tienen alto valor nutricional por sus contenidos de proteínas, carbohidratos, fibra, calcio y hierro, entre otros (Yanaguchi, 1983). El brócoli fresco es altamente perecedero, pero su vida de anaquel se puede prolongar por tres a cuatro semanas si se almacena a $0{ }^{\circ} \mathrm{C}$ (Serrano et al., 2006; Makhlouf et al., 1989) mientras que a $20{ }^{\circ} \mathrm{C}$ sólo dura de 2 a $3 \mathrm{~d}$ (Wang, 1977). El estrés de la cosecha es particularmente severo en los órganos que contienen tejidos inmaduros que estaban en crecimiento al momento de la cosecha (King y Morris, 1994), porque son incapaces de mantener una homeostasis metabólica y rápidamente senecen (Huber, 1987). El deterioro del brócoli se manifiesta principalmente por la pérdida de peso, amarillamiento y endurecimiento del tallo (Serrano et al., 2006).

Para mantener la calidad del florete después de la cosecha, es esencial preenfriar lo más rápido posible, para bajar la tasa de respiración y mantener los tejidos turgentes (Brennan y Shewfelt, 1989). Las mayores pérdidas de azúcares, ácidos orgánicos y proteínas en minifloretes de brócoli almacenados a $20{ }^{\circ} \mathrm{C}$ y oscuridad, ocurren durante las primeras $6 \mathrm{~h}$ (King y Morris, 1994). 
Según Finger et al. (1999), cabezas de brócoli almacenadas a $25{ }^{\circ} \mathrm{C}$ y $96 \%$ de humedad relativa en oscuridad, pierden turgencia y valor comercial a las $48 \mathrm{~h}$ después de la cosecha. Serrano et al. (2006) encontraron que los floretes del cv. 'Marathon' almacenados a $1{ }^{\circ} \mathrm{C} \mathrm{y}$ $90 \% \mathrm{HR}$, perdieron $46.3 \pm 1.04 \%$ de peso después de 20 d. Pramanik et al. (2006) no detectaron cambios significativos en la pérdida de peso en floretes de brócoli almacenados por 7 y $14 \mathrm{~d} \mathrm{a} 1{ }^{\circ} \mathrm{C}$, pero al ser transferidos a $20{ }^{\circ} \mathrm{C}$ por $5 \mathrm{~d}$, la pérdida de peso se incrementó gradualmente en ambos y la mayor tasa de respiración se registró en los floretes previamente almacenados por $7 \mathrm{~d}$ a $1{ }^{\circ} \mathrm{C}$.

El tamaño del florete es el principal carácter comercial en el brócoli (Wescott y Callan, 1990), y la densidad de plantación es el principal factor que afecta el rendimiento cuando éste es expresado por unidad de área (Wien y Wurr, 1997). Mediante la manipulación de la densidad de plantación se puede ajustar el peso del florete a los diferentes mercados (Fernández et al., 1991). La alta densidad de plantación en este cultivo se ha relacionado con una reducción del tamaño y peso del florete, reducido número de retoños secundarios (Chung, 1982), menor diámetro de tallo, menor rendimiento por planta (Damato, 2000) y menor incidencia de tallo hueco (Zink y Ankana, 1951; Cutcliffe, 1972).

Francescangeli et al. (2006) encontraron que las densidades de 2, 4, 6 y 8 plantas $\mathrm{m}^{-2}$ no afectaban los periodos vegetativo y reproductivo, ni tampoco el número de hojas de la planta, pero sí el área foliar, la longitud del tallo, la altura de la planta, y los pesos fresco y seco del florete; las tres primeras variables se incrementaron linealmente con el incremento en la densidad de plantación, y las últimas dos disminuyeron. Albarracín et al. (1995) mencionan que el diámetro de la inflorescencia es una característica importante en el brócoli, sobre todo cuando se comercializa por paquetes (una o varias inflorescencias atadas con una cuerda), ya que a mayor diámetro se necesitan menos inflorescencias para conformar la unidad de comercialización.

La Norma Oficial Mexicana NMX-FF-046-1982 de la Secretaría de Comercio y Fomento Industrial establece que para la calidad México Extra y México No. 1, el diámetro ecuatorial del florete debe ser mayor de $7 \mathrm{~cm}$ y que se encuentre en punto sazón cuando las yemas están cerradas y la inflorescencia esté compacta. Una característica física que reduce la calidad del florete es la presencia del desorden denominado "Tallo hueco", un defecto importante para mercado en fresco, porque el tallo hueco es un hueco en el centro del tallo que se extiende por debajo del florete y queda expuesto al momento del corte (Hipp, 1974). Este desorden es más severo cuando las plantas crecen rápidamente con altos niveles de fertilización nitrogenada, clima cálido, humedad adecuada y deficiencias de B (Vigier y Cutcliffe, 1985). La proporción adecuada de N y B para cada suelo y clima, reduce la presencia de tallo hueco en el brócoli (Moniruzzaman et al., 2007).

Las evaluaciones de rendimiento y calidad de florete son esenciales para elegir cultivares que cumplan con los estándares del mercado (Sterrett et al., 2004). Además, el tiempo que transcurre entre la cosecha del brócoli y su consumo significa alteraciones en la composición del producto que pueden afectar su calidad. El objetivo de este estudio fue evaluar el efecto del cultivar y de la densidad de plantación en la calidad física del florete de brócoli.

\section{MATERIALES Y MÉTODOS}

Ocho cultivares se evaluaron bajo condiciones de riego durante el verano del 2005 y 12 durante el verano del 2006, en un campo experimental ubicado en, Apaseo el Grande, Guanajuato, México (20 $34^{\prime} 00^{\prime}$ ' LN, $100^{\circ} 50^{\prime}$ $00^{\prime \prime}$ LO, y $1765 \mathrm{msnm}$ ). Parcelas de aproximadamente 6 x $8 \mathrm{~m}$, se trasplantaron con los cvs. 'Mónaco', 'Domador', 'Heritage', 'Grandísimo', 'Marathon', 'Patrón', 'Ironman' y 'Tláloc' para el 2005, y en el 2006 se agregaron 'Legacy', 'Patriot', 'Avenger' y 'Máximo', y se eliminó 'Tláloc'. El sistema de plantación fue a doble hilera con una distancia entre surcos de $1 \mathrm{~m}$ a tres densidades de plantación $\left(55,65,75\right.$ mil plantas ha $\left.^{-1}\right)$. Las parcelas se distribuyeron en un diseño experimental de bloques al azar con dos repeticiones. El trasplante se hizo en julio y se cosechó en la primera semana de octubre, en ambos años. Antes del trasplante se aplicaron los herbicidas Prowl ${ }^{\circledR}$ (i.a. pendimetalina) y $\operatorname{Ronstar}{ }^{\circledR}$ (i.a oxadiazon) a dosis de $1.5 \mathrm{~L} \mathrm{ha}^{-1}$ de cada uno. Durante el desarrollo de la planta se aplicaron los insecticidas Pirestar ${ }^{\circledR}$ (i.a. permetrina), Polar ${ }^{\circledR}$ (i.a. metamidofos), Orthene ${ }^{\circledR}$ (i.a. acephato) y Javelin ${ }^{\circledR}$ (i.a. Bacillus thuringiensis), a dosis de $0.6 \mathrm{~L} \mathrm{ha}^{-1}, 1 \mathrm{~L} \mathrm{ha}^{-1}, 1.5 \mathrm{~kg} \mathrm{ha}^{-1}$ y $1 \mathrm{~kg} \mathrm{ha}^{-1}$, respectivamente. Se hicieron dos escardas y un deshierbe manual y la fertilización fue $380 \mathrm{~N}-60 \mathrm{P}-$ $125 \mathrm{~K}$. La precipitación promedio de julio a septiembre fue de $89.8 \mathrm{~mm}$ mensuales en $2005 \mathrm{y}$ de $138.3 \mathrm{~mm}$ mensuales en 2006; la temperatura promedio fue de $20^{\circ} \mathrm{C}$ para los mismos meses en ambos años.

\section{Variables medidas}

Para la evaluación del diámetro de cabeza y del hueco en el tallo, número de minifloretes y pérdida de peso, se 
tomaron floretes en estado de madurez comercial, con flores compactas y sin apertura floral.

Diámetro de florete y de hueco en el tallo. A la cosecha se seleccionó una muestra aleatoria de 10 floretes por parcela, y en cada florete se midió con vernier el diámetro ecuatorial $(\mathrm{cm})$ y el diámetro del hueco en el tallo, en cm (Figura 1).

Número de minifloretes. De cada parcela se tomaron cuatro floretes, los cuales se seccionaron en minifloretes de acuerdo con la estructura de cada cultivar. Los minifloretes se clasificaron por su diámetro en tres categorías grandes: $(4.5-6 \mathrm{~cm})$, medianos $(3-4 \mathrm{~cm})$ y pequeños $(1-2 \mathrm{~cm})$.

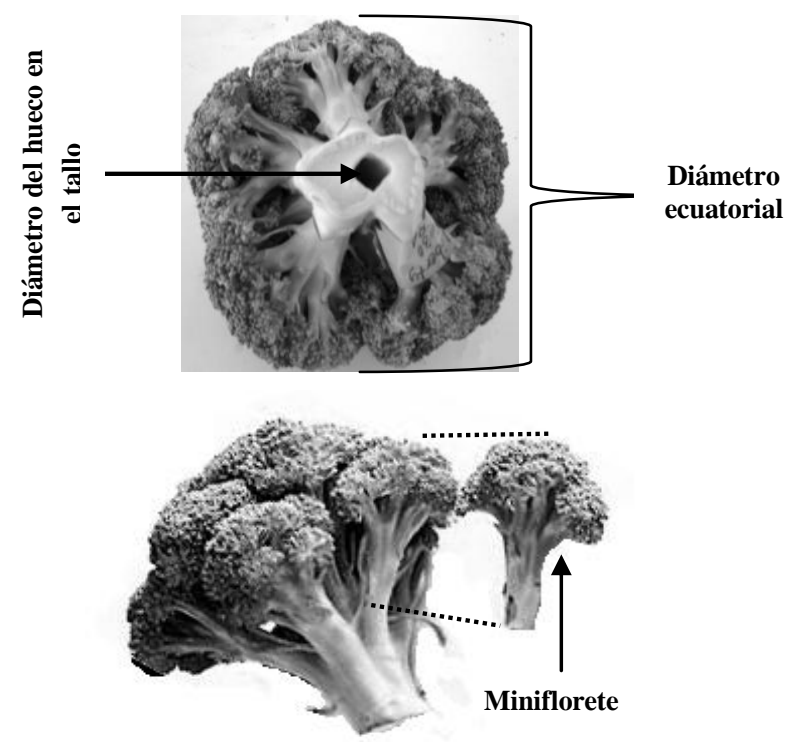

Figura1. Florete de brócoli en el que se muestran dos variables medidas.

Pérdida de peso (g). Se midió en una muestra de 10 floretes de brócoli por parcela, obtenidos de la parte central de la parcela, a fin de evitar la influencia de la orilla en el desarrollo del brócoli. Los floretes se marcaron y pesaron individualmente: al momento de la cosecha y a las $5,10,20,30$ y $40 \mathrm{~h}$ después de la cosecha (hdc). Luego los floretes se almacenaron en contenedores de plástico de $50.9 \times 35.5 \times 30.7 \mathrm{~cm}$, con ranuras en los cuatro lados para la circulación del aire, a una temperatura ambiente de 18 a $25{ }^{\circ} \mathrm{C}$, con el fin de simular las condiciones de transporte del campo a las empacadoras. Los floretes se mantuvieron en una humedad relativa promedio de $73 \%$ y con un fotoperiodo de $12 / 12 \mathrm{~h}$ de luz y oscuridad. Se utilizó una balanza portátil digital Ohaus ${ }^{\circledR}$ con capacidad de $6000 \mathrm{~g}$ y aproximación de $1 \mathrm{~g}$ (Ohaus Corp. Florham Park, NJ, USA.). La pérdida de peso acumulada se expresó como porcentaje del peso inicial.

\section{Análisis estadístico}

A los datos de cada año se les aplicó un análisis de varianza (SAS versión 8.1) para determinar el efecto de la densidad y de los cultivares sobre las variables de calidad del fruto. Se utilizó la prueba de medias de Tukey para determinar diferencias entre tratamientos.

\section{RESULTADOS Y DISCUSIÓN}

\section{Diámetro de florete}

En los dos años estudiados (2005 y 2006) el diámetro de florete fue inversamente proporcional a la densidad de plantación, ya que a mayor densidad fue menor el diámetro (Cuadro 1). Este fenómeno también fue reportado por Chung (1982) y Damato (2000). Lo anterior es un aspecto positivo para las empresas procesadoras de brócoli, ya que requieren menor número de cortes para el procesamiento de floretes.

El efecto del cultivar en el diámetro del florete fue significativo en ambos años $(\mathrm{P} \leq 0.05)$. De los siete cultivares evaluados tanto en el 2005 como en el 2006, el que registró el mayor diámetro de florete fue 'Mónaco' con 19.9 cm (Cuadro 2). En 2005, los cvs. 'Mónaco' y 'Patrón' registraron el mayor diámetro de florete, 17.7 cm mientras que el cv; 'Ironman' registró el menor diámetro, $14.8 \mathrm{~cm}$ (Cuadro 3). La interacción entre densidades y cultivares no tuvo efecto $(\mathrm{P} \leq 0.05)$ en el diámetro de florete, en el 2005. Para el 2006, los cvs. 'Mónaco' y 'Máximo' fueron iguales entre sí, y superiores $(\mathrm{P} \leq 0.05)$ a las demás variedades con un diámetro de $22.1 \mathrm{~cm}$; el menor diámetro lo tuvo el cv. 'Grandísimo' con 17.3 cm (Cuadro 4). En 2006 la interacción densidad y cultivar tuvo efecto significativo ( $\mathrm{P}$ $\leq 0.05$ ), debido a que los cvs. 'Mónaco' y 'Heritage' a una densidad de 55 mil plantas ha $^{-1}$ registraron los promedios más altos (22.7 y $22.6 \mathrm{~cm}$, respectivamente), mientras que el florete más pequeño $(15.4 \mathrm{~cm})$ lo registró la combinación del cv. 'Patriot' con 65 mil plantas ha' ${ }^{1}$ (Cuadro 4).

El hecho de que el cv. 'Mónaco' haya registrado el mayor diámetro de florete en ambos años, puede ser importante para aumentar el rendimiento por unidad de área sin tener que reducir la densidad de plantación, cuando el mercado requiere floretes grandes. Según Albarracín et al. (1995), los cultivares con inflorescencias de mayor diámetro tienen el potencial de generar mayor ingreso por unidad de área. 
Cuadro 1. Efecto de la densidad de plantación en las variables de calidad evaluadas en el 2005 y 2006 en Apaseo el Grande, Guanajuato, México.

\begin{tabular}{lcccccccc}
\hline $\begin{array}{l}\text { Densidad } \\
\text { plantas ha }\end{array}$ & \multicolumn{2}{l}{ Pérdida de peso a las $40 \mathrm{hdc}(\%)$} & \multicolumn{2}{c}{ Diámetro del hueco en el tallo (cm) } & \multicolumn{2}{c}{ Diámetro de florete (cm) } & Número de minifloretes \\
\hline & 2005 & 2006 & 2005 & 2006 & 2005 & 2006 & 2005 & 2006 \\
\hline 55000 & $13.2 \mathrm{ab}$ & $14.7 \mathrm{a}$ & $1.1 \mathrm{a}$ & $1.8 \mathrm{a}$ & $17.1 \mathrm{a}$ & $21.0 \mathrm{a}$ & $8.7 \mathrm{a}$ & $8.3 \mathrm{a}$ \\
65000 & $13.1 \mathrm{~b}$ & $13.2 \mathrm{~b}$ & $0.9 \mathrm{ab}$ & $1.7 \mathrm{a}$ & $16.4 \mathrm{ab}$ & $20.1 \mathrm{~b}$ & $8.5 \mathrm{a}$ & $7.9 \mathrm{a}$ \\
75000 & $14.3 \mathrm{a}$ & $14.4 \mathrm{a}$ & $0.7 \mathrm{~b}$ & $1.5 \mathrm{~b}$ & $15.8 \mathrm{~b}$ & $19.6 \mathrm{~b}$ & $7.7 \mathrm{a}$ & $7.7 \mathrm{a}$ \\
\hline
\end{tabular}

Medias con la misma letra en una columna, no difieren estadísticamente (Tukey, 0.05).

Cuadro 2. Comparaciones múltiples de medias de siete variedades para cuatro variables de calidad de florete en promedio de los años 2005 y 2006, en Apaseo el Grande, Guanajuato, México.

\begin{tabular}{lcccc}
\hline Cultivar & $\begin{array}{c}\text { Pérdida de peso a las } \\
40 \mathrm{hdc}(\%)\end{array}$ & $\begin{array}{c}\text { Diámetro del hueco en el } \\
\text { tallo }(\mathrm{cm})\end{array}$ & $\begin{array}{c}\text { Diámetro de florete } \\
(\mathrm{cm})\end{array}$ & $\begin{array}{c}\text { Número de } \\
\text { minifloretes }\end{array}$ \\
\hline 'Domador' & $12.7 \mathrm{a}$ & $0.8 \mathrm{bc}$ & $18.2 \mathrm{bc}$ & $8.3 \mathrm{a}$ \\
'Marathon' & $12.9 \mathrm{a}$ & $0.7 \mathrm{bc}$ & $18.4 \mathrm{ab}$ & $7.5 \mathrm{a}$ \\
'Heritage' & $13.5 \mathrm{a}$ & $1.0 \mathrm{a}$ & $18.7 \mathrm{ab}$ & $8.4 \mathrm{a}$ \\
'Mónaco' & $13.6 \mathrm{a}$ & $0.9 \mathrm{ab}$ & $19.9 \mathrm{a}$ & $8.2 \mathrm{a}$ \\
'Ironman' & $14.6 \mathrm{a}$ & $0.4 \mathrm{~d}$ & $18.1 \mathrm{bc}$ & $7.1 \mathrm{a}$ \\
'Grandísimo' & $14.6 \mathrm{a}$ & $0.9 \mathrm{bc}$ & $17.0 \mathrm{c}$ & $9.3 \mathrm{a}$ \\
'Patrón' & $14.7 \mathrm{a}$ & $0.7 \mathrm{c}$ & $19.1 \mathrm{ab}$ & $8.3 \mathrm{a}$ \\
\hline
\end{tabular}

Medias con la misma letra en una columna, no difieren estadísticamente (Tukey, 0.05).

Cuadro 3. Comparaciones múltiples de medias de variedades en las variables de calidad evaluadas en el 2005 en Apaseo el Grande, Guanajuato, México.

\begin{tabular}{lcccc}
\hline Cultivar & $\begin{array}{c}\text { Pérdida de peso a las } \\
40 \text { hdc }(\%)\end{array}$ & $\begin{array}{c}\text { Diámetro del hueco } \\
\text { en el tallo }(\mathrm{cm})\end{array}$ & $\begin{array}{c}\text { Diámetro de } \\
\text { florete }(\mathrm{cm})\end{array}$ & $\begin{array}{c}\text { Número de } \\
\text { minifloretes }\end{array}$ \\
\hline 'Ironman' & $15.1 \mathrm{a}$ & $0.5 \mathrm{c}$ & $14.8 \mathrm{~b}$ & $5.5 \mathrm{~b}$ \\
'Heritage' & $10.0 \mathrm{a}$ & $1.1 \mathrm{ab}$ & $16.2 \mathrm{ab}$ & $8.8 \mathrm{ab}$ \\
'Patrón' & $13.8 \mathrm{a}$ & $0.6 \mathrm{bc}$ & $17.7 \mathrm{a}$ & $9.3 \mathrm{ab}$ \\
'Marathon' & $13.5 \mathrm{a}$ & $1.0 \mathrm{ab}$ & $17.2 \mathrm{ab}$ & $8.3 \mathrm{ab}$ \\
'Grandísimo' & $13.3 \mathrm{a}$ & $1.0 \mathrm{ab}$ & $16.8 \mathrm{ab}$ & $11.0 \mathrm{a}$ \\
'Mónaco' & $12.9 \mathrm{a}$ & $1.2 \mathrm{a}$ & $17.7 \mathrm{a}$ & $8.4 \mathrm{ab}$ \\
'Domador' & $12.9 \mathrm{a}$ & $1.1 \mathrm{ab}$ & $15.5 \mathrm{ab}$ & $8.0 \mathrm{ab}$ \\
'Tláloc' & $12.7 \mathrm{a}$ & $1.2 \mathrm{a}$ & $15.8 \mathrm{ab}$ & $7.3 \mathrm{ab}$ \\
\hline
\end{tabular}

Entre columnas, las medias con la misma letra en una columna, no difieren estadísticamente (Tukey, 0.05).

Cuadro 4. Comparaciones múltiples de medias de variedades en las variables de calidad evaluadas en el 2006 en Apaseo el Grande, Guanajuato, México.

\begin{tabular}{lcccc}
\hline Cultivar & $\begin{array}{c}\text { Pérdida de peso a las } 40 \\
\text { hdc (\%) }\end{array}$ & $\begin{array}{c}\text { Diámetro del hueco } \\
\text { en el tallo }(\mathrm{cm})\end{array}$ & $\begin{array}{c}\text { Diámetro de } \\
\text { florete }(\mathrm{cm})\end{array}$ & $\begin{array}{c}\text { Número de } \\
\text { minifloretes }\end{array}$ \\
\hline 'Marathon' & $12.4 \mathrm{c}$ & $1.4 \mathrm{c}$ & $19.7 \mathrm{c}$ & $6.6 \mathrm{ab}$ \\
'Legacy' & $12.6 \mathrm{c}$ & $1.8 \mathrm{ab}$ & $20.5 \mathrm{~b}$ & $8.1 \mathrm{ab}$ \\
'Domador' & $12.7 \mathrm{c}$ & $1.6 \mathrm{bc}$ & $20.9 \mathrm{ab}$ & $8.6 \mathrm{ab}$ \\
'Máximo' & $12.7 \mathrm{c}$ & $2.2 \mathrm{a}$ & $22.1 \mathrm{a}$ & $8.3 \mathrm{ab}$ \\
'Heritage' & $13.1 \mathrm{c}$ & $1.9 \mathrm{ab}$ & $21.2 \mathrm{ab}$ & $8.0 \mathrm{ab}$ \\
'Ironman' & $14.1 \mathrm{bc}$ & $0.9 \mathrm{~d}$ & $21.3 \mathrm{ab}$ & $8.7 \mathrm{ab}$ \\
'Monaco' & $14.3 \mathrm{bc}$ & $1.7 \mathrm{bc}$ & $22.1 \mathrm{a}$ & $8.0 \mathrm{ab}$ \\
'Liberty' & $14.4 \mathrm{bc}$ & $1.7 \mathrm{bc}$ & $20.5 \mathrm{~b}$ & $8.6 \mathrm{ab}$ \\
'Avenger' & $14.5 \mathrm{bc}$ & $1.8 \mathrm{ab}$ & $18.7 \mathrm{~cd}$ & $9.2 \mathrm{a}$ \\
'Patrón' & $15.6 \mathrm{ab}$ & $1.4 \mathrm{c}$ & $20.5 \mathrm{~b}$ & $7.3 \mathrm{ab}$ \\
'Grandísimo' & $15.8 \mathrm{ab}$ & $1.7 \mathrm{bc}$ & $17.3 \mathrm{e}$ & $7.7 \mathrm{ab}$ \\
'Patriot' & $16.9 \mathrm{a}$ & $1.4 \mathrm{c}$ & $17.6 \mathrm{de}$ & $6.5 \mathrm{ab}$ \\
\hline
\end{tabular}

Entre columnas, las medias con la misma letra en una columna, no difieren estadísticamente (Tukey, 0.05). 


\section{Diámetro del hueco en el tallo}

La densidad de plantación, tanto en 2005 como en 2006, tuvo efectos significativos $(\mathrm{P} \leq 0.05)$ en el diámetro del hueco en el tallo. El diámetro del hueco también fue inversamente proporcional a la densidad, pues a mayor densidad se observó menor diámetro del hueco (Cuadro 1). A 75000 plantas ha ${ }^{-1}$ se registró el menor diámetro del hueco en el tallo $(1.5 \mathrm{~cm})$. Estos resultados concuerdan con los de Cutcliffe (1972) y Hipp (1974), quienes reportaron que a mayor densidad de plantación había menor incidencia de "tallo hueco". Lo anterior tal vez se debe a que la absorción de $\mathrm{N}$ por la planta fue más lenta $\mathrm{o}$ menor en alta competencia poblacional que reduce la tasa de crecimiento del florete y así favorece a obtener un menor diámetro del hueco (Moniruzzaman et al., 2007). Según Hipp (1974), es factible reducir la presencia del hueco en el tallo mediante alargamiento del ciclo de crecimiento a más de $110 \mathrm{~d}$ de trasplante a cosecha. En el estudio que aquí se reporta los ciclos fueron de $80 \mathrm{~d}$ aproximadamente, mostrando presencia de tallo hueco en todos los cultivares.

El efecto del cultivar en el diámetro de hueco en el tallo fue significativo $(P \leq 0.05)$ en 2005 y 2006. En el 2005 los cvs. 'Mónaco' y 'Tláloc' tuvieron un diámetro de hueco $>1.2 \mathrm{~cm}$, que supero al del cv. 'Ironman' con 0.5 cm (Cuadro 3). En 2006 el cv. 'Máximo' tuvo el mayor hueco $(2.2 \mathrm{~cm})$, mientras que 'Ironman' presentó el menor $(0.9 \mathrm{~cm})$ (Cuadro 4).

La interacción densidad cultivar tuvo efecto significativo $(\mathrm{P} \leq 0.05)$ en 2005 y 2006. En 2005 los cvs. 'Tláloc', 'Domador', 'Mónaco' y 'Heritage' crecidos a una densidad de 55000 plantas ha $^{-1}$ produjeron los mayores diámetros del hueco, con 1.41, 1.37, 1.35 y 1.2 $\mathrm{cm}$ respectivamente (Cuadro 3 ). El cultivar que produjo el menor diámetro de hueco $(0.05 \mathrm{~cm})$ fue 'Ironman' a una densidad de 75000 plantas ha ${ }^{-1}$. Para el 2006, el cv. 'Máximo' crecido a una densidad de 65000 plantas ha $^{-1}$ registró el mayor diámetro del hueco $(2.2 \mathrm{~cm})$ e 'Ironman' a las densidades de 75000 y 65000 plantas ha $^{-1}$ tuvo el menor diámetro $(0.7 \mathrm{~cm})$.

Para lograr reducir el tallo hueco se deben combinar varios factores, como la densidad de plantación, la variedad y la fertilización. En cuanto a esta última, Moniruzzaman et al. (2007) afirman que al combinar 1.5 $\mathrm{kg} \mathrm{B} \mathrm{ha}{ }^{-1}+100 \mathrm{~kg} \mathrm{~N} \mathrm{ha}^{-1}$ se disminuye la presencia del hueco en el tallo. En el presente estudio el cv. 'Ironman' fue el más tolerante al desorden del hueco, y en los dos años de estudio produjo el menor hueco. Esto es importante cuando se destina el florete al mercado fresco, ya que como menciona Hipp (1974), es un defecto visible que le resta calidad al brócoli.

Tanto el diámetro de cabeza como el diámetro del hueco en el tallo fueron mayores $(\mathrm{P} \leq 0.05)$ en los materiales cultivados en el 2006 que en el 2005 (Cuadro 1). Posiblemente la diferencia entre años se debe a que durante los meses cultivo (julio a septiembre) del 2006 la precipitación en la región fue de $138.3 \mathrm{~mm}$ mensuales, mientras que en los mismos meses del 2005 fue de 89.8 $\mathrm{mm}$ mensuales; es decir, la mayor precipitación del año 2006 pudo haber inducido un mayor desarrollo de las plantas, así como mayores diámetros de florete y de tallo hueco.

\section{Número de minifloretes}

Entre los años 2005 y 2006 no hubo diferencias ( $\mathrm{P} \leq$ 0.05 ) en número de minifloretes, y la densidad y cultivar tampoco presentaron diferencias $(\mathrm{P} \leq 0.05)$ en las siete variedades evaluadas en ambos años (Cuadro 2). En 2005 el cv. 'Grandísimo' produjo el mayor número de minifloretes (11), mientras que el cv. 'Ironman' presentó menor número (6) (Cuadro 3), de los ocho evaluados. En 2005 no hubo diferencia $(\mathrm{P} \leq 0.05)$ en los números de minifloretes grandes, medianos y pequeños cuyo promedio fue de 8 por cabeza (Cuadro 3).

De los 12 cultivares evaluados en 2006 'Avenger' dio el mayor número (9) de minifloretes y 'Patriot' el menor (7), (Cuadro 4). Los minifloretes grandes fueron ( $\mathrm{P} \leq$ 0.05 ) mas abundantes (13 por cabeza), que los medianos y pequeños (6 y 5 , respectivamente).

El hecho de que en el 2006 se hayan producido más minifloretes grandes, se atribuye a que en este mismo año se obtuvo también mayor diámetro del florete, atribuible a una mayor precipitación durante el periodo de cultivo; al respecto, la luz, la temperatura y la lluvia juegan un papel importante en el crecimiento y rendimiento del brócoli (Ahmed y Siddique, 2004).

\section{Pérdida de peso}

Los siete cultivares evaluados en 2005 y 2006 no presentaron diferencias $(\mathrm{P} \leq 0.05)$ en pérdida de peso (Cuadro 2), y en promedio las tres densidades de plantación mostraron una reducción progresiva del peso a través de $40 \mathrm{hdc}$, posiblemente porque las temperaturas de 18 a $25{ }^{\circ} \mathrm{C}$ y la humedad relativa de $73 \%$ causaron deshidratación. Al respecto, Finger et al. (1999) mencionan que las temperaturas de $25{ }^{\circ} \mathrm{C}$ causan pérdida de turgencia (firmeza) en los tejidos del brócoli almacenado. En el presente estudio los floretes mostraron 
disminución de turgencia y de valor comercial cuando la pérdida de peso llegó a $5 \%$; los porcentajes de pérdida peso a las 40 hdc fueron de 10 a $16.9 \%$ (Cuadros 3 y 4), con los cuales perdieron su valor comercial debido a la flacidez de sus tejidos. Toivonen (1997) también reportó pérdidas de peso en brócoli almacenado a $13{ }^{\circ} \mathrm{C}$ y $95 \%$ de HR, tanto en floretes cubiertos como no cubiertos con película microperforada. Según Pramanik et al. (2004), en brócoli almacenado durante $5 \mathrm{~d}$ la pérdida de peso es mayor durante las primeras $24 \mathrm{~h}$ de almacenamiento pero los síntomas de senescencia se presentan hasta 3 y 4 ddc.

La densidad tuvo un efecto significativo $(\mathrm{P} \leq 0.05)$ en el porcentaje de pérdida de peso en 2005 y 2006. En 2005, los floretes procedentes de la densidad de 75000 plantas ha ${ }^{-1}$ registraron la mayor pérdida de peso (14.3\%) a las $40 \mathrm{hdc}$, seguida de 55000 plantas ha $^{-1}(13.2 \%)$ y la densidad en la que registró la menor pérdida de peso fue la de 65000 plantas ha $^{-1}$ con $13.1 \%$ (Cuadro 1). Para el 2006, las densidades de 75000 y de 55000 plantas ha ${ }^{-1}$ fueron iguales en pérdida de peso, con promedios de 14.6 y $14.4 \%$ respectivamente, mientras que la de 65000 plantas ha ${ }^{-1}$ tuvo menor pérdida de peso $(13.2 \%)$.

En términos generales, las densidades de 75000 y 55 000 plantas $\mathrm{ha}^{-1}$ produjeron floretes que perdieron mayor porcentaje de peso, lo cual parece estar asociado con los correspondientes diámetros de florete. En 55000 plantas $\mathrm{ha}^{-1}$ se obtuvieron diámetros de floretes mayores, de modo que la superficie de exposición al ambiente es mayor y así tendrían mayor deshidratación. En 75000 plantas ha $^{-1}$ los floretes fueron pequeños en la superficie de exposición y en peso, por lo que el porcentaje pérdida de peso fue alto.

Entre cultivares no hubo dependencias $(\mathrm{P} \leq 0.05)$ en el porcentaje de pérdida de peso para el 2005 (Cuadro 3), mientras que en 2006 el factor cultivar mostró efecto significativo $(\mathrm{P} \leq 0.05)$ en pérdida de peso. El cultivar con mayor pérdida de peso fue 'Patriot' con $16.9 \%$, y los de menores porcentajes fueron 'Marathon' (12.4\%), 'Legacy' (12.6 \%), 'Domador' (12.7 \%), 'Máximo' (12.7 $\%)$ y 'Heritage' (13.1 \%) (Cuadro 4). En general en los cultivares que produjeron floretes pequeños las pérdidas de peso porcentuales eran mayores. Las pérdidas de peso también estuvieron influenciadas por la arquitectura del florete, ya que los floretes con un domo compacto fueron menos propensos a la pérdida de peso, atribuible a su menor área expuesta al ambiente.

Las combinaciones que registraron menores pérdidas de peso durante 2005 fueron 'Domador' a 65000 plantas ha ${ }^{-1}$ (12\%) y 'Tláloc' a 55000 plantas ha $^{-1}$ (11.8\%). En 2006 el cultivar que registró la menor pérdida de peso fue 'Máximo' a una densidad de 65000 plantas ha ${ }^{-1}$.
La pérdida de peso significa pérdidas económicas en la cadena de comercialización. Para evitar la deshidratación y mantener una buena calidad del brócoli otros autores han recomendado almacenar en atmosferas modificadas, y someter los floretes a hidroenfriamiento y bajas temperaturas (Toivonen, 1997; Carvalho y Clemente, 2004; Serrano et al., 2006).

\section{CONCLUSIONES}

Los diámetros de florete y de hueco en el tallo fueron menores $(\mathrm{P} \leq 0.05)$ en los procedentes de la densidad de 75000 plantas $\mathrm{ha}^{-1}$, mientras que en la densidad de 55000 plantas ha ${ }^{-1}$ presentó los mayores diámetros de florete y de hueco en el tallo. El cv. 'Mónaco' registró el mayor diámetro de florete $(\mathrm{P} \leq 0.05)$ al momento de la cosecha, en contraste con el cv. 'Ironman' que presentó el menor diámetro de hueco. El número de minifloretes no fue influenciado por la densidad de plantación, pero sí por el cultivar; 'Grandísimo' y 'Avenger' presentaron la mayor cantidad de minifloretes en 2005 y 2006. La menor pérdida de peso (13.1\%) se registró en floretes de brócoli procedentes de la densidad de 65000 plantas ha $^{-1}$.

\section{AGRADECIMIENTOS}

A la empresa SEMINIS-Estación de Investigación Bajío, por el apoyo brindado durante el establecimiento y desarrollo del experimento.

\section{BIBLIOGRAFÍA}

Albarracín M, C Berbin, W Machado (1995) Evaluación agronómica de cultivares de brócoli (Brassica oleracea var. italica). Rev. Fac. Agron. (Maracay) 21:71-83.

Ahmed M J, W Siddique (2004) Effect of sowing dates on growth and yield of broccoli (Brassica oleracea L.) under rawalakot conditions. Asian J. Plant Sci. 3:167-169.

Brennan P S, R L Shewfelt (1989) Effect of cooling delay at harvest on broccoli quality during postharvest storage. J. Food Qual. 12:13-22.

Carvalho P de T, E Clemente (2004) The influence of the broccoli (Brassica oleracea var. itálica) fill weigth on postharvest quality. Ciencia Tecnol. Alim. Campinas 24:646-651.

Chung B (1982) Effects of plant density on the maturity and once over harvest yields of broccoli. J. Hort. Sci. 57:365-372.

Cutcliffe J A (1972) Effects of plant spacing and nitrogen on incidence of hollow stem in broccoli. Can. J. Plant Sci. 52:833-834.

Damato G (2000) Late sowing dates and high plant density in six cultivars of broccoli for processing. Acta Hort. 533:267-274.

Fernández J A, A González, S Bañón, A García (1991) La producción de brócoli: influencia de la densidad de plantación y de la disposición de las plantas. Hortofruticultura 10:58-64.

Finger F L, L Endres, P R Mosquim, M Puiatti (1999) Physiological changes during postharvest senescence of broccoli. Pesq. Agropec. Brasil. 34:1565-1569.

Francescangeli N, M A Sangiacomo, H Martí (2006) Effects of plant density in broccoli on yield and radiation use efficiency. Sci. Hort. 110:135-143. 
Hipp B W (1974) Influence of nitrogen and maturity rate on hollow stem in broccoli. Sci. Hort. 9:68-69.

Huber D J (1987) Postharvest senescence: an introduction to the symposium. HortScience 22:853-854.

King G A, S C Morris (1994) Early compositional changes during postharvest senescence of broccoli. J. Amer. Soc. Hort. Sci. 119:1000-1005.

Makhlouf J, C Willemot, J Arul, F Castaigne, J-P Emond (1989) Long-term storage of broccoli under controlled atmosphere. HortScience 24:637-639.

Moniruzzaman M, S M L Rahman, M G Kibria, M A Rahman, M M Hossain (2007) Effect of boron and nitrogen on yield and hollowstem of broccoli. J. Soil Nat. 1:24-29.

Pramanik B K, T Matsui, H Suzuki, Y Kosugi (2004) Changes in activities of sucrose synthase and sucrose phosphate synthase and sugar content during postharvest senescence in two broccoli cultivars. Asian J. Plant Sci. 3:398-402.

Pramanik B K, T Matsui, H Suzuki, Y Kosugi (2006) Compositional and some enzymatic changes relating to sugar metabolism in broccoli during storage at $1{ }^{\circ} \mathrm{C}$ and subsequent senescence at $20^{\circ} \mathrm{C}$. Acta Hort. 706:219-227.

Serrano M, D Martínez-Romero, F Guillén, S Castillo, D Valero (2006) Maintenance of broccoli quality and functional properties during cold storage as affected by modified atmosphere packaging. Postharv. Biol. Technol. 39:61-68.
Sterrett S B, K G Haynes, C P Savage Jr (2004) Cluster analyses on quality attributes identify broccoli cultivars suitable for early and main-season harvests on the eastern shore of Virginia. HorTechnology 14:376-380.

Toivonen P M A (1997) The effects of storage temperature, storage duration, hydro-cooling, and micro-perforated wrap on shelf life of broccoli (Brassica oleracea L., itálica group). Postharv. Biol. Technol. 10:59-65.

Vigier B, J A Cutcliffe (1985) Influence of boron and nitrogen rateo $n$ incidence of hollow stem in broccoli. Can. J. Plant Sci. 65:421424.

Wang C Y (1977) Effect of aminoethoxy analog of rhizobitoxine and sodium benzoate on senescence of broccoli. HortScience 12:54 56.

Wescott H C, N W Callan (1990) Modeling plant population and rectangularity effects on broccoli head wights and yield. J. Amer. Soc. Hort. Sci. 115:893-897.

Wien H D, D C E Wurr (1997) Cauliflower, broccoli, cabbage and Brussels sprouts. In: The Physiology of Vegetable Crops. H C Wien (ed). CAB International. New York, USA. pp:511-552 .

Yanaguchi M (1983) World Vegetable Principles, Production and Nutritive values. AVI Publishing Company, INC. Wet Port, Connection, USA. $405 \mathrm{p}$.

Zink F W, D A Ankana (1951) The effect of spacing on the growth of sprouting broccoli. Proc. Amer. Soc. Hort. Sci. 58:160-164. 